\section{A PROMOÇÃO DASAÚDE FAMILIAL NO QUOTIDIANO DA ATENÇÃO PRIMÁRIA: UMA REVISÃO INTEGRATIVA}

\author{
The Promotion of Familial Health In The Everyday Life Of \\ Primary Care: An Integrative Review
}

\section{RESUMO}

Objetivo: Este estudo tem como objetivo conhecer e analisar a produção do conhecimento sobre a promoção da saúde familial desenvolvida pela enfermagem nos últimos 10 anos. Métodos: Trata-se de uma revisão integrativa de literatura, nas bases de dados LILACS (Literatura Latino-Americana em Ciências da Saúde), IBECS (Índice Bibliográfico Espanhol de Ciências da Saúde) e MEDLINE (Medical Literature Analysis and Retrieval System Online), utilizando os descritores Promoção da Saúde, Enfermagem Familiar, Atenção Primária à Saúde. Foram selecionados artigos completos de pesquisa, revisões integrativas e relatos de experiências nos idiomas português, inglês e espanhol, no período de 2004 a 2014, totalizando 281 artigos, posteriormente, prosseguiu-se a um total de 12 artigos, considerando o objetivo deste trabalho. Resultados: A produção do conhecimento sobre a Promoção da Saúde Familial desenvolvida pela Enfermagem nos últimos 10 anos é limitada em todo mundo. O Enfermeiro de família é o profissional que está mais próximo do contexto familiar, social e comunitário, em relação às outras especialidades da enfermagem e o cuidado para a Promoção da Saúde perpassa todos os estágios do ciclo de vida familiar. Porém, apenas um estudo define o que é o enfermeiro de família e é apontada críticas às normas e políticas de saúde no Brasil dentro da atenção primária à saúde. Conclusão: A Promoção da Saúde Familial dentro do contexto da Atenção Primária à Saúde é uma área emergente no Brasil e no mundo e vem ganhando espaço dentro das políticas públicas de saúde, levando a reorientação do modelo de atenção à saúde. O Enfermeiro no quotidiano da atenção primária executa diferentes ações, em diversos cenários como escola, comunidade e serviço, porém é preciso investir mais na sua formação e qualificação, adotando-se métodos de ensino participativos, incentivando a criação de instrumentos que mostrem como o Enfermeiro tem trabalhado, possibilitando a valorização e o reconhecimento deste profissional.

Descritores: Enfermagem familiar; Promoção da Saúde; Atenção Primária; Atividades Cotidianas.

\footnotetext{
ABSTRACT

Objective: This article objective is to know and analyze the production of knowledge on the promotion of family health in a primary care basis developed by nursing area in the last 10 years. Methods: This is an integrative literature review, in the databases LILACS (Latin American Literature in Health Sciences), IBECS (Spanish Bibliographical Index of Health Sciences) and MEDLINE (Medical Literature analysis and Retrieval System Online), using the keywords Health promotion, Family Nursing, Primary Health Care. It was selected full research articles, integrative reviews and experience reports in Portuguese, English and Spanish, in the 2004-2014 period, totaling 281 articles, from those, 12 articles have been selected considering the main objective of this research. Results: The production of knowledge about the Family Health Promotion developed by Nursing in the last 10 years is limited worldwide. The Family Nurse is the professional who is closest to the family, social and community context in relation to other nursing specialties and care for Health Promotion permeates all stages of the family life cycle. However, only one study defines what the family nurse is and critics are pointed out to the norms and health policies in Brazil within primary health care. Conclusion: The promotion of Familial Health within the context of Primary
}

Artigo de Revisão
1) Universidade Federal de Santa Catarina UFSC - Florianópolis (SC) - Brasil
Este artigo seguiu as normas e formatação estabelecidas pelo $5^{\circ} \mathrm{CIAIQ}$ - Congresso Ibero-Americano em Investigação Qualitativa 
Health Care is an emerging area in Brazil and the world, and has been gaining space within public health policies, leading to a reorientation of the health care model. The nurse in the everyday life of primary care performs different actions in different school settings, community and services, but more investment is needed in their training and qualification, adopting participatory teaching methods, encouraging the creation of instruments that show how the nurse has worked, allowing the appreciation and recognition of this professional.

Descriptors: Family Nursing; Health Promotion; Primary Care; Activities of Daily Living

\section{INTRODUÇÃO}

A família como foco de cuidado da equipe da Estratégia de Saúde da Família (ESF) é uma área a ser explorada nos estudos científicos brasileiros, e um desafio para o desenvolvimento das ações e cuidado para a Promoção da Saúde dos profissionais da área.

A retomada do papel da família, nas políticas e programas de saúde em diversos países do mundo, pode ser vista mais expressivamente após a Carta de Ottawa, no ano de 1986. Este documento, resultado da I Conferência Internacional sobre Promoção da Saúde em Ottawa, Canadá, fundamentado na ótica da Promoção da Saúde, chamou a atenção para o papel das políticas públicas, e a inclusão dos diferentes atores que fazem parte deste processo de construção dos cuidados, incluindo o setor saúde, setores sociais e econômicos, organizações voluntárias e não governamentais, autoridades locais, indústria, mídia e as pessoas em todas as esferas da vida (pessoa, família e

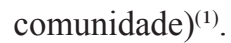

A Carta de Ottawa define promoção da saúde como o processo de capacitação das pessoas, famílias e comunidades para que tenham autonomia sob suas escolhas e uma melhor qualidade de vida no seu processo de viver. Ela percorre todos os níveis de atenção à saúde possibilitando mudanças no modo de pensar e agir as questões de envolvem a saúde ${ }^{(2)}$.

A Carta de Ottawa trouxe um conceito de Promoção da Saúde mais abrangente mostrando que vários fatores influenciam na saúde das pessoas, famílias e comunidades, para além de um estilo de vida saudável e extrapola o setor, para um bem-estar global, considerando também os determinantes sociais e ambientais onde as pessoas, famílias e comunidade estão inseridas. Desta forma, o documento foi motivo de constantes debates teóricos e conceituais que permanecem até hoje, e o mantém como o principal ideário norteador para as políticas governamentais para a área da saúde( ${ }^{(3)}$.
No Brasil, as principais ações de Promoção da Saúde da família têm sido trabalhadas na Atenção Primária à Saúde (APS) integrada às ESF, que é a principal porta de entrada do usuário ao Sistema Único de Saúde (SUS) ${ }^{(4)}$.

A Estratégia de Saúde da Família (ESF) emergiu em 1994, como Programa de Saúde da Família (PSF), caracterizando-se como uma estratégia prioritária da AP para a reorientação dos serviços de saúde de acordo com os preceito e diretrizes do SUS. Com a proposta de romper com o cuidado biomédico e fragmentando, visando um cuidado integral, centrado na saúde das famílias, ampliando a resolubilidade, o acesso aos serviços, compreendendo as especificidades e situação de saúde de cada comunidade atendida ${ }^{(4)}$.

O Enfermeiro da ESF é o profissional que coordena a equipe de Agentes Comunitários de Saúde (ACS) e Técnicos de Enfermagem, capacitando-os para um cuidado integral e humanizado; promover espaços de educação permanente; dar assistência às pessoas e suas famílias na área de abrangência, em todas as fases do seu ciclo vital, atentando para os princípios da integralidade, equidade e universalidade do acesso ao serviço, além de protagonizar relações entre as equipes e com os demais setores e serviços ${ }^{(4)}$.

Assim, o Enfermeiro tem sido o profissional de referência neste campo de atuação, e o principal articulador no processo de mudança do modelo de atenção à saúde, pois está presente no dia a dia do serviço, atuando na assistência e na gerência do cuidado, prosseguindo para além de práticas individuais, contemplando as reais necessidades das famílias a partir do contexto que estão inseridas.

Tendo em vista a relevância da temática citada, este estudo tem como objetivo: conhecer e analisar a produção do conhecimento sobre a promoção da saúde familial desenvolvida pela enfermagem nos últimos 10 anos.

\section{MÉTODOS}

Trata-se de uma revisão integrativa de literatura acerca da promoção da saúde familial realizada pela Enfermagem. A revisão integrativa vem contribuir com o aprofundamento do conhecimento sobre as produções científicas relativas a uma determinada temática e consiste na construção de uma análise ampla da literatura, para discussões sobre conceitos, revisão de teorias, métodos e resultados de pesquisas, assim como reflexões sobre a realização de futuros estudos, o que contribui para a análise dos estudos realizados na área da saúde e enfermagem ${ }^{(5)}$.

Visando contemplar o objetivo de "conhecer e analisar a produção do conhecimento sobre a promoção da saúde familial desenvolvida pela enfermagem nos últimos 10 anos", buscou-se responder a seguinte questão: Como está 
a produção do conhecimento acerca da promoção da saúde familial desenvolvida nos estudos da enfermagem nos últimos 10 anos?

Para seleção dos artigos foram usadas as bases de dados LILACS (Literatura Latino-Americana em Ciências da Saúde), IBECS (Índice Bibliográfico Espanhol de Ciências da Saúde) e MEDLINE (Medical Literature Analysis and Retrieval System Online), adotando-se os descritores DECS (Descritores em Ciências da Saúde) e MeSH (Medical Subject Headings).

No dia 19 de novembro de 2015, foi realizada a busca na base MEDLINE e no dia 05 de janeiro de 2016 foi realizada a busca nas bases LILACS e IBECS, adotandose os seguintes descritores: family nurse OR family health OR care family OR family practice OR family centered care OR family centered AND health promotion OR health promotion intervention $\mathrm{OR}$ health promotion interventions AND primary care OR primary health OR primary healthcare OR primary health system $\mathrm{OR}$ primary health service.

A amostra do estudo foi realizada a partir dos critérios de inclusão na pesquisa: estudos disponíveis no editor científico Bireme/BVS e PUBMED, contemplando as bases de dados LILACS, IBECS, MEDLINE, que contenham os descritores DECS e MeSH, publicados em periódicos na forma completa (fulltext), tais como: artigos de pesquisa, revisões integrativas ou relatos de experiências, relacionados à temática nos idiomas português, inglês e espanhol, nos anos de 2004 até 2014. Os critérios de exclusão foram: editoriais, cartas, artigos de opinião, comentários, resumo de anais, ensaios, notas prévias, publicações duplicadas, teses e dissertações, manuais, artigos completos que não estão dispostos na íntegra, documentos oficiais de programas nacionais e internacionais, livros, materiais publicados em outros idiomas que não sejam inglês, espanhol, português.

A análise dos estudos encontrados foi sistematizada, seguindo as etapas da pesquisa bibliográfica ${ }^{(6)}$ contemplando: o levantamento bibliográfico preliminar nas bases de dados supracitadas; a leitura exploratória dos estudos, verificando a viabilidade dos estudos encontrados para a revisão da literatura; a leitura seletiva, analisando, de maneira específica, a pertinência dos estudos; a leitura analítica, sumarizando as informações encontradas de maneira crítica; a leitura interpretativa, articulando os conhecimentos versados em todos os estudos analisados; e a elaboração do texto final que sintetiza os resultados da pesquisa da literatura.

A partir da leitura interpretativa da análise foi possível identificar duas categorias: O Papel do Enfermeiro de Família e sua formação para a Promoção da Saúde, e a Metodologias para Promoção da Saúde familial nos diferentes cenários na prática de enfermagem.

\section{RESULTADOS E DISCUSSÃO}

$\mathrm{Na}$ primeira etapa, foram encontrados 281 artigos, posteriormente, prosseguiu-se a leitura minuciosa dos resumos, buscando determinar os critérios de inclusão e exclusão, a adequação e relevância dos assuntos abordados, considerando o objetivo deste trabalho, chegando-se a um total de 12 artigos.

O Quadro I apresenta os artigos selecionados, junto com seus autores, ano de publicação, periódico em que foi publicado, bem como a base de dados em que foi encontrado $\mathrm{o}$ artigo.

Os artigos analisados foram publicados entre os anos de 2007 a 2014, não aparecendo publicações entre 2004 a 2006, sendo que as publicações seguem uma incidência constante variando de uma a duas em cada ano. Dentre os periódicos apresentados, a Revista da Escola de Enfermagem da Universidade de São Paulo (EEUSP) apresentou o maior número de publicações $(\mathrm{n}=2)$, sendo que os demais apresentaram uma publicação por periódico.

Os artigos analisados são originados de diversos países: sendo 34\% $(\mathrm{n}=4)$ do Brasil, 17\% $(\mathrm{n}=2)$ dos Estados Unidos, $17 \%(\mathrm{n}=2)$ da Austrália, $8 \%(\mathrm{n}=1)$ da Nova Zelândia, $8 \%(\mathrm{n}=1)$ do Canadá, $8 \%(\mathrm{n}=1)$ da Coréia e $8 \%(n=1)$ do Irã, apontado uma temática que vem sendo estudada a nível mundial.

Os artigos publicados por instituições brasileiras são quatro: Escola de Enfermagem da Universidade de São Paulo (EEUSP) (dois), Universidade Estadual de Campinas (um) e Universidade de Franca (UNIFRAN) (um), ressaltando que todas as instituições estão localizadas no estado de São Paulo (SP), região sudeste do país.

Quanto aos tipos de estudo, 59\% $(\mathrm{n}=7)$ são caracterizados como pesquisa qualitativa; $25 \%(\mathrm{n}=3)$ são estudos quantitativos; $8 \%(\mathrm{n}=1)$ é uma revisão de literatura; e $8 \%(n=1)$ é de caráter quantitativo - qualitativo.

Em relação aos sujeitos dos estudos da temática da promoção da saúde familial na enfermagem apresentaramse diversificados, a maioria mulheres e suas famílias $25 \%$ $(\mathrm{n}=3)$, seguido de Enfermeiros, cuidador de criança, adolescentes, familiares de pessoas idosas, familiares de crianças com deficiências, estudantes de enfermagem, e pacientes dependentes de substâncias psicoativas e suas famílias.

\section{O papel do Enfermeiro de família e sua formação para a promoção da saúde}

A definição do Enfermeiro de família foi encontrada em apenas um estudo de origem europeia, onde este profissional realiza a prestação de cuidados primários à saúde, possibilitando prioritariamente o acesso aos serviços 
Quadro I - Artigos sobre a Promoção da Saúde da Família incluídos na revisão integrativa.

\begin{tabular}{|c|c|c|c|c|c|}
\hline $\mathbf{N}^{0}$ & Título & Autores & Ano & Revista & Fonte \\
\hline 1 & $\begin{array}{l}\text { Meaning and action in employed mothers' } \\
\text { health work. }\end{array}$ & Kushner, K. E. & 2007 & $\begin{array}{l}\text { Journal of Family } \\
\text { Nursing }\end{array}$ & $\begin{array}{l}\text { MEDLINE/ } \\
\text { PubMed }\end{array}$ \\
\hline 2 & $\begin{array}{l}\text { The role of nursing in governmentality, } \\
\text { biopower and population health: family health } \\
\text { nursing. }\end{array}$ & Thompson, L. & 2007 & Health \&Place & $\begin{array}{l}\text { MEDLINE/ } \\
\text { PubMed }\end{array}$ \\
\hline 3 & $\begin{array}{l}\text { Evaluation of an educational booklet about } \\
\text { childcare promotion from the family's } \\
\text { perception regarding health and citizenship }\end{array}$ & $\begin{array}{l}\text { Grippo, M. L., } \\
\text { Fracolli, L A. }\end{array}$ & 2008 & $\begin{array}{c}\text { Revista da Escola } \\
\text { de Enfermagem } \\
\text { da USP }\end{array}$ & $\begin{array}{l}\text { MEDLINE/ } \\
\text { PubMed }\end{array}$ \\
\hline 4 & $\begin{array}{l}\text { Social support and networks in health promotion } \\
\text { of older people: a case study in Brazil }\end{array}$ & $\begin{array}{l}\text { Rocha, S. M, } \\
\text { Nogueira, M. L., } \\
\text { Cesario. M. }\end{array}$ & 2009 & $\begin{array}{l}\text { International } \\
\text { Journal of Older } \\
\text { People Nursing }\end{array}$ & $\begin{array}{l}\text { MEDLINE/ } \\
\text { PubMed }\end{array}$ \\
\hline 5 & $\begin{array}{l}\text { Functional clinical placements: a driver for } \\
\text { change. }\end{array}$ & $\begin{array}{l}\text { Goddard, L., } \\
\text { Mackey, S., } \\
\text { Davidson, P. M. }\end{array}$ & 2010 & $\begin{array}{l}\text { Nurse Education } \\
\text { Today }\end{array}$ & $\begin{array}{l}\text { MEDLINE/ } \\
\text { PubMed }\end{array}$ \\
\hline 6 & $\begin{array}{l}\text { Health promotion and related factors among } \\
\text { korean goose mothers. }\end{array}$ & Cha, C. & 2010 & $\frac{\text { Asian Nursing }}{\text { Research }}$ & $\begin{array}{l}\text { MEDLINE/ } \\
\text { PubMed }\end{array}$ \\
\hline 7 & $\begin{array}{l}\text { A abordagem à família na Estratégia Saúde da } \\
\text { Família: uma revisão integrativa da literatura. }\end{array}$ & $\begin{array}{l}\text { Silva, M. C. L. } \\
\text { S. R.; Silva, L.; } \\
\text { Bousso, R. S. }\end{array}$ & 2011 & $\begin{array}{c}\text { Revista da Escola } \\
\text { de Enfermagem } \\
\text { da USP }\end{array}$ & LILACS \\
\hline 8 & $\begin{array}{l}\text { Teachers as researchers: a narrative pedagogical } \\
\text { approach to transforming a graduate family and } \\
\text { health promotion course }\end{array}$ & Brykczynski, K. A. & 2012 & $\begin{array}{l}\text { Nursing } \\
\text { Education } \\
\text { Perspectives }\end{array}$ & $\begin{array}{l}\text { MEDLINE/ } \\
\text { PubMed }\end{array}$ \\
\hline 9 & $\begin{array}{l}\text { Experience of adolescents in an activity of } \\
\text { health promotion }\end{array}$ & $\begin{array}{l}\text { Ferreira, J. A. R., } \\
\text { Barros E. M.; Sousa } \\
\text { R. A., Souza, L. J. }\end{array}$ & 2013 & $\begin{array}{c}\text { Revista Brasileira } \\
\text { de Enfermagem }\end{array}$ & $\begin{array}{l}\text { MEDLINE/ } \\
\text { PubMed }\end{array}$ \\
\hline 10 & $\begin{array}{l}\text { Impact of a nurse-directed, coordinated school } \\
\text { health program to enhance physical activity } \\
\text { behaviors and reduce body mass index among } \\
\text { minority children: a parallel-group, randomized } \\
\text { control Trial }\end{array}$ & $\begin{array}{l}\text { Wright, K., Giger, } \\
\text { J.N., Norris, K., } \\
\text { Suro, Z. }\end{array}$ & 2013 & $\begin{array}{c}\text { International } \\
\text { Journal of } \\
\text { Nursing Studies }\end{array}$ & $\begin{array}{l}\text { MEDLINE/ } \\
\text { PubMed }\end{array}$ \\
\hline 11 & $\begin{array}{l}\text { Be our guest': challenges and benefits of using } \\
\text { 'family conversations' to collect qualitative } \\
\text { data about infant feeding and parenting. }\end{array}$ & $\begin{array}{l}\text { Reid J, Schmied } \\
\text { V, Sheehan A, } \\
\text { Fenwick J. }\end{array}$ & 2014 & $\begin{array}{c}\text { Journal of } \\
\text { Clinical Nursing }\end{array}$ & $\begin{array}{l}\text { MEDLINE/ } \\
\text { PubMed }\end{array}$ \\
\hline 12 & $\begin{array}{l}\text { Effects of Educational Intervention on Health- } \\
\text { Promoting Lifestyle and Health-Related Life } \\
\text { quality of Methamphetamine Users and Their } \\
\text { Families: a Randomized Clinical Trial. }\end{array}$ & $\begin{array}{l}\text { Ghasemi A, } \\
\text { Estebsari F, } \\
\text { Bastaminia A, } \\
\text { Jamshidi E, } \\
\text { Dastoorpoor M. }\end{array}$ & 2014 & $\begin{array}{c}\text { Iranian Red } \\
\text { Crescent Medical } \\
\text { Journal }\end{array}$ & $\begin{array}{l}\text { MEDLINE/ } \\
\text { PubMed }\end{array}$ \\
\hline
\end{tabular}

Fonte: Base de Dados da BVS e Pubmed: MEDLINE, LILACS e IBECS, 2014 e 2015.

em áreas carentes e nos locais mais pobres da região que atua, apontando ainda que o Enfermeiro de família está mais próximo do contexto familiar, social e comunitário, em relação às outras especialidades da enfermagem. Ainda ressalta que o Enfermeiro de família tem como instrumento de avaliação da família e suas relações o genograma e ecomapa $^{(7)}$.

Em relação aos estudos brasileiros, constatou-se que os documentos normativos das políticas públicas de saúde que abordam a família nas ESF não apresentam como esta prática deve acontecer e nem como é feito o preparo dos profissionais para o trabalho com famílias na APS, além de apontar a prática do Enfermeiro pautada em um modelo de atenção individual e curativo ${ }^{(8)}$.

Analisando a formação do Enfermeiro para a promoção da saúde familial não foi encontrado nenhum estudo brasileiro com esta temática. Dentre os estudos, um estudo americano realizado com professores e alunos em uma 
disciplina de pós-graduação retratou o processo de melhoria da disciplina que aborda a temática da promoção da saúde com um método ativo e participativo. Ao final da pesquisa, foi apontada a dificuldade dos professores em conciliar o ensino focado na promoção da saúde, com o objetivo de aprendizagem dos alunos de enfermagem, uma vez que possuíam uma visão biomédica da saúde individual não identificando a família como um todo. Porém, com uso do método ativo e participativo, foi possível trabalhar estas dificuldades e melhorar a avaliação da disciplina e do curso, mostrando a importância de estudar e aprender outros métodos de trabalho para a promoção da saúde $\mathrm{e}^{(9)}$.

Vale ainda destacar que desde 1990 a Organização Mundial da Saúde já definia o papel do Enfermeiro de família como um profissional de cuidados de saúde primários que desenvolvem ações de promoção da saúde e prevenção de doenças, além de trabalhar intensamente com as pessoas em suas casas, oferecer cuidados para um estilo de vida sadável, detectar fatores de risco e problemas de saúde e realizar apoio social ${ }^{(10)}$.

\section{Metodologias para Promoção da Saúde familial nos diferentes cenários na prática de enfermagem}

Quanto aos aspectos metodológicos adotados nas pesquisas, considerando tanto a abordagem quantitativa, como a qualitativa, foi possível perceber o seguinte panorama:

Dentre os estudos quantitativos, um estudo utilizou um grupo controle com adolescentes e seus familiares para avaliar o impacto de um programa de saúde escolar coordenado e dirigido por Enfermeiro para melhorar comportamentos de atividade física e reduzir o índice de massa corporal entre crianças ${ }^{(11)}$.

Outro estudo analisou as "mães gansos" coreanas (termo utilizado para as mães que migram para outros países por um período de tempo prolongado para promover educação aos seus filhos, porém seguem casadas com seus esposos que ainda residem na Coreia) na qual foram investigado seus comportamentos de promoção da saúde na área da América do Norte. Utilizou-se para coleta um questionário demográfico criado pelo autor e a Health Promotion Lifestyle Profile (HPLP) que mede as frequências dos comportamentos de promoção da saúde em seis áreas: auto realização, responsabilidade para saúde, atividade física, nutrição, relação interpessoal, e gestão do stress $^{(12)}$.

O ensaio clínico randomizado, realizado com usuários de metanfetamina em recuperação e membros da família, utilizou para sua coleta de dados o Modelo de Promoção da Saúde de Pender (PHPM) e o Modelo de Empoderamento Centrado na Família (FCEM) para avaliar uma intervenção educativa $^{(13)}$.
Os estudos quantitativos apontam maior prevalência de pesquisas para promoção da saúde com foco na mudança de comportamento, como hábitos alimentares, atividade física, controle de estresse, não ampliando a promoção da saúde para além de um estilo de vida saudável.

Dentre os estudos qualitativos, percebe-se um olhar mais ampliado para as questões da promoção da saúde familial. Um estudo analisou os significados da saúde familial e da saúde pessoal de mulheres assalariadas com filhos menores de 18 anos, utilizou como referencial teórico metodológico a estratégia de Triangulação Teórica da Teoria Crítica, Feminismo e Interacionismo Simbólico ${ }^{(14)}$.

Um segundo estudo abordou famílias de pessoas com mais de 70 anos, com objetivo de compreender a vida das pessoas idosas e suas famílias, e explorar possibilidades de cuidado tendo em vista a promoção da saúde, utilizou como instrumento de coleta de dados o genograma, para conhecer as famílias e suas interações, e o ecomapa, para conhecer o contexto das relações familiares com a comunidade e os pontos fortes delas com as organizações e instituições. Assim, reforça o uso do genograma e do ecomapa como instrumentos de trabalho tanto na assistência quanto na pesquisa da enfermagem, pois possibilita avaliar o contexto familiar e criar possibilibidades de mudanças ${ }^{(15)}$.

A pesquisa - ação foi usada para desenvolver uma intervenção de promoção de saúde da família para melhorar a saúde e o bem-estar dos membros da família e reduzir o risco para a doença ${ }^{(16)}$. Outro estudo trabalhou com um grupo de vivencias com adolescentes e seus familiares, abordando práticas de promoção da saúde no pré-natal utilizando peças teatrais ${ }^{(17)}$.

A pesquisa-ação tem sido utilizada em diversos estudos com a temática da promoção da saúde com o objetivo do "aprender fazendo", é uma investigação com e para as pessoas, possibilitando a participação ativa, redução das relações hierárquicas, e contribui para a promoção da equidade no processo de tomada de decisão. Ela vem sendo utilizada na promoção da saúde para desenvolver intervenções comunitárias complexas, envolvendo "pessoas comuns" que se identificam não só as suas necessidades de saúde, mas os seus direitos e questões em torno do cuidado $^{(16)}$.

Outro método utilizado para coleta de dados foram as "conversas familiares" para explorar o impacto potencial que a rede social de amigos e familiares de mães primigestas tem sobre a experiência de parentalidade e a introdução da alimentação infantil ${ }^{(17)}$.

Dentre os estudos caracterizados como quantitativos e qualitativos, foi encontrado um estudo com crianças de 0 a 59 meses e seus familiares, tendo como objetivos: contribuir na avaliação da efetividade da Cartilha: "Toda Hora é Hora de 
Cuidar"; analisar a percepção dos familiares sobre os temas discutidos pela cartilha; identificar a aceitação da cartilha pela população atendida; caracterizar a compreensão pelos familiares sobre os conceitos que subjazem os temas das Cartilhas. Este estudo utilizou para coleta de dados um formulário com perguntas abertas para análise qualitativa, e perguntas fechadas para a análise quantitativa, adotando ainda o software Statistical Package for the Social Sciences - SPSS para organização e análise dos dados ${ }^{(18)}$.

No panorama dos estudos qualitativos é possível observar o predomínio de técnicas e instrumentos com participação ativa dos sujeitos numa dialética que possibilite compreender as questões da promoção da saúde familial com olhar ampliado para as questões de saúde.

Este cenário de publicações aponta que a Promoção da Saúde familial vem sendo trabalhada com os diferentes atores envolvidos no processo do cuidado da atenção primária, na perspectiva de informar as pessoas para terem uma melhor saúde familial, através de um processo interativo que valoriza a individualidade dos sujeitos ${ }^{(16)}$.

Os estudos também buscam resgatar as dimensões da promoção da saúde vividas no dia a dia da família como a cultura, os fatores sociais, as atividades que envolvem mudança de comportamento como atividade física e nutrição, além das relações interpessoais, apontando quão complexo e variado são os cuidados para a promoção da saúde ${ }^{(12)}$.

Deste modo, a Promoção da Saúde da Família envolve ações de saúde para e com a família, a partir das experiências do seu dia a dia e da construção e manutenção de rotinas. Assim, a Promoção da Saúde da Família se dá no contexto da vida cotidiana, sendo uma forma de viver que engloba oportunidades continuas e enfrentamento no dia a $\operatorname{dia}^{(14)}$.

No Brasil, o envolvimento das famílias nas ações desenvolvidas pelas ESF precisam ser incentivados e incorporados nas rotinas dos serviços, ressaltando que para trabalhar com promoção da saúde "é preciso adoção de atitudes positivas pela sociedade, e nessa linha de raciocínio, a literatura advoga o empowerment em saúde" 19:431 , considerando a adoção de intervenções eficazes para solucionar os problemas de saúde pública.

Observamos que o cuidado para a Promoção da Saúde perpassa todos os estágios do ciclo de vida familiar, considerando a qualidade da assistência oferecida para cada grupo, na qual, a participação do familiar, nas atividades desenvolvidas pela enfermagem mostra-se positiva como, por exemplo, a adesão às consultas e aos tratamentos de doenças. Assim, o familiar que participa do processo fortalece as relações com o individuo que precisa do cuidado $^{(13)}$.
Apesar de apenas um artigo definir Promoção da Saúde, conforme a definição da União Internacional de Promoção e Educação para a Saúde (IUHPE), como o "processo que capacita comunidades, centrado na equidade, e considera essencial a participação da população nas ações de promoção à saúde, reconhecendo e potencializando o conhecimento desta comunidade."19:431, outro estudo, apresentou a definição de Promoção da Saúde da Família, como diferente de prevenção das doenças, ela é um conjunto de fatores com foco no aumento do bem-estar e qualidade de vida da família ${ }^{(15)}$.

Os demais estudos trazem apenas o termo Promoção da Saúde como essencial dentro das ações e cuidados de enfermagem, além de apontarem a Promoção da Saúde como uma estratégia para o cuidado à família.

\section{CONCLUSÃO}

Esta revisão integrativa indica que a produção do conhecimento sobre a Promoção da Saúde Familial desenvolvida pela Enfermagem nos últimos 10 anos é limitada, ou seja, os estudos são escassos, somando apenas 12 do total que atendem ao objetivo do estudo. A lacuna de produção na área existente entre 2004 e 2007, bem como a ausência de estudos realizados em outras regiões do Brasil, especialmente, Nordeste e no Sul, que tem uma tradição de trabalhos pioneiros e reconhecidos publicados na área da enfermagem familial.

No Brasil, as políticas públicas de saúde sofreram um grande avanço após a reforma sanitária e criação do SUS, porém é preciso adaptá-las aos diferentes cenários e contextos que os profissionais, famílias e comunidade estão inseridas para torná-las efetivas na prática.

Quando analisado as metodologias dos estudos concluiu-se que os estudos quantitativos apontam maior prevalência de pesquisas para promoção da saúde com foco na mudança de comportamento, como hábitos alimentares, atividade física, controle de estresse, não trazendo a promoção da saúde para além de um estilo de vida saudável. Já os estudos qualitativos prevalecem um olhar mais ampliado para as questões da promoção da saúde familial trabalhando com os significados das relações e do dia a dia das famílias com técnicas participativas no processo de construção dos estudos.

O enfermeiro inserido APS realiza um cuidado para a promoção da saúde que perpassa todas das fases do ciclo de vida familiar e se dá na vida cotidiana, nas relações interpessoais, nas diferentes culturas, nos cenários sociais e no modo como as famílias vivem. Assim, estando mais próximo das famílias e comunidade tem a oportunidade de envolver as famílias nos processos construtivos do seu 
dia a dia, por meio de métodos de ensino participativos e incentivando um viver mais saudável.

\section{REFERÊNCIAS}

1. Gutierrez DMD, Minayo MCS. Produção de conhecimento sobre cuidados da saúde no âmbito da família. Ciência \& Saúde Coletiva. 2010; 15 (1):1497-1508. Disponível em: <http://www. scielo.br/scielo.php?script=sci_arttext\&pid $=$ S1413-81232010000700062>.

2. WHO. Ottawa charter for health promotion. Ottawa: WHO, 1986. Disponível em: <http://www.who.int/ healthpromotion/conferences/previous/ottawa/en/ >. Acesso em: 11 jun. 2015.

3. Heidemann ITSB et al. Promoção da saúde e qualidade de vida: concepções da Carta de Ottawa em produção científica. Ciência, saúde e cuidado. 2012; 3 (11):613619. Disponível em: $<$ http://ojs.uem.br/ojs/index.php/ CiencCuidSaude/article/view/13554/pdf>.

4. Brasil. Ministério da Saúde. Portaria $n^{0} 2.488$, de 21 de outubro de 2011. Aprova a Política Nacional de Atenção Básica, estabelecendo a revisão de diretrizes e normas para a organização da Atenção Básica, para a Estratégia Saúde da Família (ESF) e o Programa de Agentes Comunitários de Saúde (PACS). Disponível em: http://bvsms.saude.gov.br/bvs/saudelegis/gm/2011 /prt2488_21_10_2011.html>.

5. Mendes KDS, Silveira RCCP, Galvão CM. Revisão integrativa: método de pesquisa para a incorporação de evidências na saúde e na enfermagem. Texto e Contexto Enfermagem. 2008; 17(4):758-64. Disponível em: http://www.scielo.br/scielo.php?script=sci_arttext\&pi $\mathrm{d}=\mathrm{S} 0104-07072008000400018$ > .

6. Ganog LH. Integrative reviews of nursing research. Res Nurs Health. 1987 feb; 10(1):1-11. DOI: 10.1002/ nur.4770100103.

7. Thompson L. The role of nursing in governmentality, biopower and population health: family health nursing. Health \& Place. 2007; 14(2008):76-84. Disponível em: http://www.sciencedirect.com/science/article/pii/ S1353829207000342>.

8. Silva MCLSR, Silva L, Bousso RS. A abordagem à família na Estratégia Saúde da Família: uma revisão integrativa da literatura. Revista da Escola de Enfermagem da USP. 2011; 45(5):1250-5. Disponível em: http://www.scielo.br/pdf/reeusp/v45n5/v45n5a31. pdfs.
9. Brykczynski KA. Teachers as researchers: a narrative pedagogical approach to transforming a graduate family and health promotion course. 2012; 33(4):2248. Disponível em: http://eds.a.ebscohost.com/eds/ pdfviewer/pdfviewer?sid=e7a0a8c1-720d-48c7$910 \mathrm{a}-391 \mathrm{~b} 142 \mathrm{e} 85 \mathrm{f} 5 \% 40$ sessionmgr4002\&vid $=0 \& \mathrm{~h}$ $\mathrm{id}=4208>$.

10. WHO. Health21: an introduction to the health for all policy framework for the WHO European region. 1998. World Health Organisation, Regional Office for Europe, Series No. 5, Copenhagen.Disponível em: http://www. euro.who.int/_data/assets/pdf_file/0004/109759/ EHFA5-E.pdf $>$.

11. Wright $\mathrm{K}$ el al. Impact of a nurse-directed, coordinated school health program to enhance physical activity behaviors and reduce body mass index among minority children: a parallel-group, randomized control trial. Int J Nurs Stud. 2013; 50(6):727-37. Disponível em: http:// www.ncbi.nlm.nih.gov/pubmed/23021318>.

12. Cha C. Health promotion and related factors among korean goose mothers. Asian Nursing Research. 2010; 4(4):205-15. Disponível em: http://www.sciencedirect. com/science/article/pii/S1976131711600054>.

13. Ghasemi A, Estebsari F, Bastaminia A, Dastoorpoor M. Effects of Educational Intervention on HealthPromoting Lifestyle and Health-Related Life Quality of Methamphetamine Users and Their Families: A Randomized Clinical Trial. Iranian Red Crescent Medical Journal. 2014; 11(16):1-8. Disponível em: http://www.ncbi.nlm.nih.gov/pmc/articles/PMC4 329963/>.

14. Kushner KE. Meaning and action in employed mothers' health work. Journalof Family Nursing. 2007; 13(1):33-55. Disponível em: http://jfn.sagepub.com/ content/13/1/33.long $>$.

15. Rocha SM, Nogueira ML, Cesário M. Social support and networks in health promotion of older people: a case study in Brazil. International Journal Older People Nursing. 2009; 4:288-98. Disponível em: http://onlinelibrary.wiley.com/doi/10.1111/j.17483743.2009.00177.x/epdf>.

16. Goddard L, Mackey S, Davidson PM. Functional clinical placements: a driver for change. Nurse Education Today. 2010; 30(2010):398-404. Disponível em: http://www.sciencedirect.com/science/article/pii/ S0260691709001865>.

17. Ferreira Júnior AR, Barros EMA, Sousa RA, Souza LJE. Experience of adolescents in an activity of health 
promotion. Rev. bras. enferm. 2013; 66(4):611-14. Disponível em: http://www.scielo.br/pdf/reben/v66n4/ v66n4a23.pdf $>$.

18. Reid J, Schmied V, Sheehan A, Fenwick J. Be our guest': challenges and benefits of using 'family conversations' to collect qualitative data about infant feeding and parenting. Journal of Clinical Nursing. 2014; 23:2404-13. Disponível em: http://dx.doi. org/10.1111/jocn.12224>.

19. Grippo ML, Fracolli LA. Evaluation of an educational booklet about childcare promotion from the family's perception regarding health and citizenship. Revista Escola de Enfermagem da USP. 2008; 42(3):430-43. Disponível em: http:// www.scielo.br/scielo.php?script $=$ sci_arttext\&pid $=$ S0080-62342008000300003 $>$.

\section{Endereço para correspondência:}

Juliana Chaves Costa

Av. Prefeito José Juvenal Mafra, nº 6925, ap 204

Bairro: Gravatá

CEP: 88372-506 - Navegantes - Santa Catarina - Brasil

E-mail: julianachavescosta@gmail.com 